\title{
Philosophiques
}

\section{Sur la preuve de consistance de Gauthier et le programme de Frege}

\section{David DeVidi}

Volume 31, numéro 1, printemps 2004

Poincaré et la théorie de la connaissance

URI : https://id.erudit.org/iderudit/008941ar

DOI : https://doi.org/10.7202/008941ar

Aller au sommaire du numéro

Éditeur(s)

Société de philosophie du Québec

ISSN

0316-2923 (imprimé)

1492-1391 (numérique)

Découvrir la revue

Citer cet article

DeVidi, D. (2004). Sur la preuve de consistance de Gauthier et le programme de Frege. Philosophiques, 31(1), 215-220. https://doi.org/10.7202/008941ar d'utilisation que vous pouvez consulter en ligne.

https://apropos.erudit.org/fr/usagers/politique-dutilisation/ 


\title{
Sur la preuve de consistance de Gauthier et le programme de Frege
}

\author{
DAVID DEVIDI \\ University of Waterloo \\ ddevidi@uwaterloo.ca
}

Quand Philosophiques m'a donné l'occasion de commenter Internal Logic de Yvon Gauthier, j'étais très heureux - entre autres parce que j'avais l'occasion d'obtenir une copie d'un livre écrit par une figure redoutable de la philosophie des mathématiques au Canada. Après lecture cependant, j'ai pensé que Gauthier et Kluwer avaient conspiré pour produire un livre plutôt décevant.

La preuve de consistance pour ce qu'il appelle «l'arithmétique de Fermat " comme thème central du livre constitue une des sources majeures de déception. Pour plusieurs raisons, la preuve est extrêmement difficile à suivre.

Une de ces raisons est qu'on aurait souhaité que Gauthier prenne le temps de corriger quelques maladresses avant la publication de l'ouvrage - par exemple, il présente sa logique comme une forme particulière de calcul séquentiel tout en soutenant que "Since our system is a system of local logic (with minimalist and intuitionist properties), in practice we can consider only sequents $\Gamma$ where $\Delta$ consists of a unique formula" (p. 85). Malheureusement, les règles séquentielles ne sont pas fixées sous cette forme mais toujours sous une forme générale permettant plusieurs formules arbitraires comme conséquents. Pourquoi ne pas avoir pris la peine de présenter adéquatement le système à l'étude?

Les autres raisons sont plus importantes. Une de celles-là concerne la tendance de Gauthier à soutenir que la notation s'explique d'elle-même. C'est là une hypothèse hasardeuse qui fait en sorte que les publications en logique apparaissent souvent terriblement pédantes aux non-logiciens. J'ai lu la définition que Gauthier donne de la fonction d'assignation (p. 88) à plusieurs reprises et je la trouve toujours aussi opaque. Ceci s'explique entre autres par le fait que la définition introduit soudainement, sans explication, une notation que nous n'avons vue nulle part auparavant dans le livre et dont la légitimité n'est pas immédiatement manifeste. De même, lorsque nous passons à l'étape de l' "élimination des constantes logiques ", nous sommes invités à réécrire les règles du calcul séquentiel en remplaçant les composantes de la séquence par les polynômes appropriés. Mais ce qui nous manque, c'est une explication arithmétique adéquate de la ligne séparant une séquence de la suivante. La seule explication qui nous est présentée est «that the line has the meaning simply of an ordered sequence of sequents (consisting of the sequences of formulas themselves. " Cela 
peut paraître bizarre, mais je ne trouve pas cette explication éclairante. Vraisemblablement, ce qui est requis, c'est que les transitions d'une séquence à la suivante correspondent à des transformations arithmétiques légitimes mais qui ont aussi des propriétés appropriées pour modéliser la notion de «following from».

On pourrait s'attendre à des éclaircissements là-dessus en se tournant vers les résultats métalogiques que Gauthier avance pour sa théorie des modèles. C'est-à-dire que lorsqu'il démontre que le sens dans lequel il présente le calcul séquentiel est adéquat pour la classe de modèles qu'il définit, on pourrait vraisemblablement être en mesure de tirer quelque chose de ce qu'il entend par la fonction d'assignation et de la manière par laquelle il souhaite que les règles séquentielles soient comprises. Malheureusement, nous n'avons aucune de ces preuves.

En fin de compte, j'avoue que je suis incapable de présenter une évaluation sensée de cette preuve parce que je ne la comprends tout simplement pas. Cela témoigne peut-être de mes propres caprices, mais je ne crois pas me tromper en affirmant que si, moi, je ne peux saisir où Gauthier veut en venir dans ce chapitre, c'est qu'il y a de sérieux problèmes dans sa présentation - soit que la matière est formulée de façon obscure, soit que le nombre de lecteurs potentiels de ce chapitre est extrêmement limité, et ce, même pour un traité portant sur un domaine de recherche spécialisé en philosophie. Même si cette dernière alternative était fondée, il n'en reste pas moins que l'argument aurait pu être présenté d'une façon plus accessible.

Mais puisque Gauthier est bien entendu un logicien accompli, peutêtre devrions-nous le prendre aux mots et tenir pour acquis que la preuve est correcte. Mais son ouvrage nous donne d'autres raisons de ne pas suivre cette voie. Car l'ouvrage semble avoir été confectionné avec une dose importante d'insouciance tant de la part de l'auteur que de la maison d'édition. On peut s'attendre à rencontrer quelques erreurs typographiques dans un livre, particulièrement lorsqu'il traite d'un sujet technique, mais celui-ci en contient plusieurs douzaines, sans compter les nombreuses phrases malheureuses dont le sens est complètement opaque. Assurément, une correction d'épreuves attentive aurait pu en réduire le nombre. Cela aurait peut-être pu éviter des erreurs typographiques comme le remplacement du quantificateur existentiel par «1/4» dans les premiers chapitres du livre et le remplacement du symbole tourniquet par "? " au chapitre 4. D'autres erreurs plus importantes semblent imputables à l'auteur lui-même plutôt qu'à la maison d'édition. Par exemple, à la page 1, nous constatons tant une incapacité à distinguer la loi fondamentale $V$ de Frege du principe de compréhension naif et le "fait» surprenant que $x \notin x$ est une contradiction. Quand cette loi fondamentale $V$ est à nouveau évoquée (p. 126), sa formulation est embrouillée. L'ouvrage contient de nombreux cas similaires de formulations brouillonnes 
qui ont pour conséquence de miner la crédibilité de l'auteur dans l'entreprise délicate visant à établir la consistance de l'arithmétique au sein même de l'arithmétique.

Ce qui en fait un livre décevant, plutôt qu'un livre inintéressant, c'est que, en dépit de tout ce qui vient d'être dit, Gauthier est un philosophe dont les idées sont intéressantes et importantes. Le projet fondationnel de Gauthier est aussi radical qu'intéressant (même si, encore une fois, il n'est pas toujours exprimé clairement). Si son affirmation selon laquelle le type d'arithmétique constructiviste qu'il nomme $F A$ est intrinsèquement consistante, il s'agit-là sans aucun doute d'un fait dont les conséquences philosophiques sont très importantes. Aussi, même si nous ne sommes pas prêts à le laisser isoler les antécédents, c'est une entreprise philosophique intéressante que d'essayer d'imaginer ce qu'en seraient les implications si la preuve était correcte. Une bonne partie du livre est évidemment consacrée à ce type de questions, mais sans le conditionnel. C'est à une des affirmations de Gauthier concernant les conséquences de sa preuve que je vais consacrer la suite de mes remarques. Car bien que sa preuve aura sûrement des conséquences philosophiques importantes, il y a de nombreux passages qui me laissent croire que Gauthier n'a pas réussi à les mettre au clair. Pour l'instant, j'aimerais me pencher sur ce que Gauthier a à dire sur les Fondements de l'arithmétique de Frege dans le chapitre 5 de son ouvrage.

En particulier, j'aimerais remettre en question l'affirmation étonnante de Gauthier suivant laquelle "Kronecker's polynomial arithmetic appears as the true realization of Frege's project. » Je crois qu'une telle affirmation n'est possible que si l'on interprète le projet de Frege de façon erronée. Car, comme le souligne Gauthier, la "logique interne» qu'il découvre dans l'arithmétique polynomiale de Kronecker est "with content» et «is not independent of arithmetic but shares its mode of construction, which a Kantian would probably call synthetic mode.» D'un autre côté, c'est précisément le but de l'entreprise logiciste de Frege que de démontrer que l'arithmétique est sans contenu intuitif, et donc en ce sens non synthétique. Comme le fait remarquer Demopoulos dans un article cité par Gauthier (Bell and Demopoulos, 1993), le travail de Frege appartient clairement à une longue tradition d'ouvrages consacrés à l'effort de libérer l'analyse de toute dépendance au recours à l'intuition géométrique ou cinétique. Comme c'est un raccourci commode et utile que de mettre l'emphase sur les motivations anti-kantiennes du projet (comme l'ont fait, par exemple, Alberto Coffa,1995), Demopoulos (voir aussi Dummett,1991) a clairement démontré que le programme peut être indépendamment motivé par la nécessité d'en finir avec le développement des soi-disant fonctions pathologiques. Les fonctions pathologiques sont celles qui ont des propriétés "inimaginables", comme celle d'être continues mais d'être nulle part différenciables, et dont l'existence pourrait être démontrée en utilisant les méthodes canoniques pour la manipulation des objets non pathologiques. 
Dans cette tradition, c'est le recours à l'intuition qui est à blâmer pour les attentes inappropriées de ce qu'on est en droit d'attendre de la notion de fonction, et c'est donc le recours à l'intuition qui doit être éliminé dans une théorie fondationnelle adéquate. Quoi qu'il en soit des disputes autour de l'interprétation de Frege, la plupart des interprètes estiment que l'œuvre de Frege appartient à cette tradition. Donc, si Gauthier peut néanmoins soutenir que l'objectif de Frege a été réalisé par la reconnaissance de l'autoconsistance de l'arithmétique polynomiale, je crois qu'il s'agit là d'un tournant révolutionnaire dans le commentaire sur Frege.

Malheureusement, l'argument de Gauthier pour défendre son affirmation est plutôt difficile à suivre. C'est bien connu que le projet de Frege a rencontré quelques difficultés face au paradoxe de Russell. Gauthier écrit à ce propos: "One knows that Frege will at the end take refuge in geometry as the sole source of the mathematical knowledge or the spatial and temporal continuum as the origin of the idea of the infinite. " Mais il ne semble pas accorder trop de poids à cette affirmation et ne semble donc pas s'opposer à ceux qui auraient prévu cela à travers un examen attentif des premiers travaux intéressants de Frege. Il semble plutôt admettre l'argument selon lequel Frege aurait lancé la serviette à la fin de sa vie, alors qu'il se livrait à des divagations imaginaires sur bon nombre de sujets.

Gauthier demande (p. 126): «How do we relate Frege's theory to Kronecker's programme?" Il nous sert alors une vague paraphrase de Grundlagen $(\$ 88)$ où Frege explique comment quelque chose peut être démontré comme une conséquence logique des définitions - et donc analytique en son sens et non au sens kantien - et ainsi élargir notre connaissance. Mais, mystérieusement, Gauthier affirme que «Frege does not pretend to have shown such a link to be analytical ", alors que cette affirmation semble contredire ce que dit Frege à la $\mathbb{\$} 88$ de Grundlagen: "The conclusions we draw from it extend our knowledge, and ought, therefore, on Kant's view, to be regarded as synthetic; and yet they can be proved by purely logical means, and are thus analytic. The truth is that they are contained in the definitions, but as plants are contained in their seeds, not as beams are contained in a house." Que l'on croie ou non que Frege a réussi à donner un contenu à la métaphore utilisée dans cette dernière phrase, il semble plutôt faire explicitement ce que Gauthier nie qu'il fait. Peut-être Gauthier a-t-il à l'esprit la remarque de Frege à la $\$ 90$ où il affirme: "I do not pretend to have made the analytic character of arithmetical propositions more than probable.» Mais il ressort clairement de ce qui suit que ce que veut dire ici Frege, c'est qu'il n'a pas présenté une preuve logique complètement explicite et sans faille, et qu'ainsi demeure la possibilité de contagion par "some other type of premise." Il est donc quelque peu trompeur d'affirmer que Frege "claims only that there is an internal connection between logic and arithmetic.» Parce qu'il dit bel et 
bien qu'il a «made it probable that the laws of arithmetic are analytic», ce qui revient à dire qu'il considère qu'il a de bonnes raisons de croire que les preuves qu'il présente pourraient être établies sans faille.

Gauthier poursuit: "If logic means only the consecution of linear sequences of elements, logic is arithmetic and there is no need for anything else than polynomials. » Ce que cela signifie exactement nous laisse un peu perplexe, mais nous pouvons affirmer avec assez de certitude que Frege n'aurait sans doute pas accepté l'antécédent. Nous pouvons douter que Frege ait fourni une caractérisation adéquate de ce qu'est un principe fondamental, c'est-à-dire logique — il y a bien entendu sa caractérisation des lois logiques qui «neither need nor admit proof » à la $\$ 3$ des Grundlagen, mais cela ne nous est d'aucun secours. Il ressort clairement de la discussion aux $\mathbb{S} \mathbb{S} 90$ et 91 que seules ces séquences de propositions qui constituent des arguments dans lesquels aucun contenu intuitif ne peut entrer sans être découvert, c'est-à-dire celles dans lesquelles les étapes sont en accord avec un nombre limité de principes qui peuvent garantir cette sorte de connexion entre les membres de la séquence, pourront compter comme des preuves logiquement correctes. L'affirmation de Gauthier suivant laquelle Frege aurait échoué à démontrer que les nombres naturels pourraient être considérés comme des objets logiques laisse tout aussi perplexe: "Frege's loss here is a conception of the autonomy of arithmetic within arithmetic, though it was his dearest wish.» Cette phrase est plutôt étrange puisque Frege tentait précisément de démontrer l'autonomie de l'arithmétique par rapport à l'intuition géométrique et cinétique, ce qui possiblement aurait pu être considéré comme son «voeu le plus cher» et, de toute façon, le sens de l'expression «autonomy of arithmetic within arithmetic » n'est pas très clair. En tout cas, si l'autonomie de l'arithmétique est le vœu le plus cher de Frege, on ne voit pas très bien comment l'arithmétique de Kronecker et la contamination de l'arithmétique par le contenu intuitif qui l'accompagne pourrait d'un façon ou d'une autre constituer la réalisation de ce projet.

La suite de cette section de l'ouvrage de Gauthier consacrée à Frege contient un bon nombre de propositions toutes aussi difficiles à interpréter. Le résultat est qu'il n'est pas facile de bien voir la position qu'entend défendre Gauthier lorsqu'il est question des implications de sa preuve de consistance pour le projet fondationnel de Frege. Cela est regrettable parce que certaines de ses affirmations paraissent peu plausibles et plutôt frappantes. S'il pouvait établir le bien-fondé de ses arguments et si d'autres philosophes pouvaient vérifier l'exactitude de ses propos, un progrès philosophique véritable pourrait en résulter. Tel quel, cependant, son argumentation ici, comme à beaucoup d'autres endroits dans le livre, m’apparaît trop obscure pour faire l'objet d'une évaluation ${ }^{1}$.

1. Je tiens à remercier Richard DeVidi et Tim Kenyon pour leurs précieuses suggestions. 


\section{Références}

Coffa, Alberto, "Kant, Bolzano and the Emergence of Logicism», William Demopoulos (ed.), Frege's Philosophy of Mathematics, Cambridge, Harvard University Press, 1995, p. 29-40.

Demopoulos, William and John L. Bell, «Frege's Theory of Concepts and Objects and the Interpretation of Second-order Logic ", Philosophia Mathematica, 1, 1993, p. 139-156.

Dummett, Michael, Frege: Philosophy of Mathematics, Cambridge, Harvard University Press, 1991 